\title{
NIVEL DE CONOCIMIENTOS SOBRE REANIMACION CARDIOPULMONAR EN ESTUDIANTES DE ENFERMERÍA EN UNA UNIVERSIDAD PUBLICA DEL PERÚ
}

\author{
Level of knowledge about cardiopulmonary resuscitation in nursing students of a \\ public university of Perú
}

Yuri Katherine Miranda Conislla, ${ }^{2 a,}$ Isabel Natividad Urure Velazco ${ }^{1, a, b, c}$

\author{
${ }^{1}$ Facultad de Enfermería, Universidad Nacional San Luis Gonzaga, Ica, Perú \\ ${ }^{2}$ Centro de Salud I -3 Jeberos, Alto Amazonas, Loreto, Perú. \\ a Lic. en Enfermería. ${ }^{b}$ Magister en Educación con mención en Administración y Planificación de la Educación Superior. ${ }^{\circ}$ Doctor en Ciencias \\ de la Salud
}

\section{RESUMEN}

El estudio tuvo como Objetivo: Identificar el nivel de conocimientos sobre reanimación cardiopulmonar en estudiantes de Enfermería en una Universidad Pública del Perú. Material y Métodos: Es una investigación de Tipo de descriptivo cuantitativo, de corte transversal. La muestra fue de 54 alumnos de VII ciclo de enfermería. Resultados: El 46,30\% de alumnos tienen los conocimientos deficientes sobre reanimación cardiopulmonar básico, el $44,44 \%$ tienen conocimientos regulares y el 9,26\% tienen conocimiento bueno. El $50 \%$ de alumnos tienen los conocimientos deficientes reanimación cardiopulmonar avanzado, el $46,30 \%$ tienen conocimiento regular y el 3,70\% tienen conocimiento bueno. Conclusiones: Con un 95\% de confianza los datos dan evidencia que el nivel de conocimientos sobre reanimación cardiopulmonar en estudiantes de Enfermería; es deficiente.

Palabras claves: nivel de conocimiento, reanimación cardiopulmonar, alumnos.

\section{SUMMARY}

The study aims to: Identify the level of knowledge about cardiopulmonary resuscitation in Nursing students of a public university of Perú. Material and method: It is a cross-sectional, quantitative descriptive type research. The sample consisted of 54 students from the 7th cycle of nursing. Results: $46,30 \%$ of students have poor knowledge about basic cardiopulmonary resuscitation, $44,44 \%$ have regular knowledge and 9,26\% have good knowledge. 50\% of students have poor advanced cardiopulmonary resuscitation knowledge, 46,30\% have regular knowledge and 3,70\% have good knowledge. Conclusions: With 95\% confidence, the data give evidence that the level of knowledge about cardiopulmonary resuscitation in Nursing students; is lacking.

Key words: level of knowledge, cardiopulmonary resuscitation, students.

\section{INTRODUCCIÓN}

La reanimación cardiopulmonar $(\mathrm{RCP})$ es el procedimiento de emergencia más utilizado, disminuye la mortalidad generada por enfermedades cardiovasculares, la importancia del conocimiento sobre este tema es indispensable y obligatoria para el personal de salud, porque salva vidas (1). Siendo la $(\mathrm{RCP})$ una respuesta organizada $\mathrm{y}$ secuencial al paro cardíaco (2).
Mundialmente se notifican cada año alrededor de 135 millones de muertes por causas cardiovasculares y el predominio de la enfermedad cardiovascular está en incremento. La prevalencia del paro cardiorrespiratorio extra hospitalario es entre 20 y 140 casos por cada 100,000 habitantes con una supervivencia que comprende entre $2 \%$ y $11 \%$ (1). 
Un gran número de las personas que atraviesan por un episodio de paro cardiorrespiratorio fallecen sin llegar al hospital. Por tal motivo la intervención inmediata de reanimación cardiopulmonar (RCP) es fundamental con la finalidad de incrementar las posibilidades de sobrevivir de la persona hasta que llegue la asistencia médica (1). Cada minuto que se retrasa la $\mathrm{RCP}$, las probabilidades de supervivencia disminuyen en un $10 \%$. (3)

El tener conocimiento tanto en maniobras de RCP como soporte vital básico (SVB) y avanzado (SVA) es considerado un requisito básico en el personal de salud ya que aumenta los resultados benéficos en la atención de pacientes que sufren de estas afecciones (1). Pudiendo duplicar o triplicar la posibilidad de conservar la vida de las víctimas de paro cardíaco. (4)

En relación al RCP en el profesional de salud se han hecho diversos estudios en donde se evidencia la falta de conocimientos adecuados sobre RCP como la investigación efectuada en Ecuador por Balcázar L. En 2016 reporta que el $89,3 \%$ de profesionales tienen conocimientos insatisfactorios. Señala que el personal de salud del servicio de urgencias tenía deficiencias en los conocimientos de reanimación cardiopulmonar avanzado (5).

Asimismo, estudios realizados en Perú reportan brechas aun no coberturadas acerca de los conocimientos en reanimación cardiopulmonar (RCP) como Lima por Bueno C, 2018; demuestra que el nivel de cognitivo sobre RCP básica del interno de medicina es de $64,5 \%$ tiene conocimiento medio con respecto a reanimación cardiopulmonar básica. El 64,5\% de Internos de Medicina tienen nivel de conocimiento medio sobre RCP Avanzado (6). En Puno el estudio realizado año 2017 por el autor Arapa A. reporta que el $65 \%$ de los estudiantes tienen un nivel de conocimiento inferior y 35\% regular (7). Ballón F. estudio desarrollado en Arequipa, acerca del nivel de cognitivo sobre RCP se obtuvo como resultados que un $72,6 \%$ de los internos encuestados tenían un nivel de conocimiento bajo, mientras que un nivel de conocimiento regular estaba representado por un $24,2 \%$ y un $3,2 \%$ tuvo un nivel de conocimiento bueno (8). Cárdenas J, Huamán C, estudio efectuado en Ica año 2016 concluye que el 52,2\% tenía un nivel de conocimientos medio, el $20,9 \%$ bajo nivel de conocimientos (9).

Se llega a la conclusión que los conocimientos de los alumnos son de regular a bajo y no están preparados para afrontar una situación de emergencia 0 ante una emergencia humanitaria donde todos los profesionales de salud deben brindar la atención inmediata y manejo oportuno a los problemas de salud ya sean personas con riesgo inminente de morir o sufrir severas complicaciones, para el efecto se hace necesario contar con profesionales que tengan alto nivel de conocimiento, un buen desempeño laboral y con compromiso social, atendiendo situaciones de emergencia.

Por lo anteriormente expuesto consideramos de importancia, realizar el estudio con el objetivo de identificar el nivel de Conocimiento sobre reanimación cardiopulmonar en estudiantes de Enfermería de la Universidad Nacional San Luis Gonzaga. El estudio se justifica porque hay una alta incidencia de muertes por patologías cardiológicas en nuestro país, un 18,3 millón de personas equivalente a un $40 \%$ de todas las muertes notificadas, el $10 \%$ de millones de esas muertes se produjeron por cardiopatía coronaria y $6,3 \%$ millones de los accidentes vasculares cerebrales. También es imprescindible prepararse desde que son estudiantes de pregrado, la teoría y la identificación de los signos y síntomas de un paro cardiorrespiratorio y su asistencia de un $\mathrm{RCP}$, así como también de la cadena de supervivencia, que abarca: prevención primaria, reconocimiento de las primeras señales de paro cardiorrespiratorio, atención pre-hospitalaria, hospitalaria y rehabilitación; estando definido el tiempo la pre-reanimación, 
reanimación y post reanimación y en el proceso de su aplicación: evaluación, acción y reevaluación.

\section{MATERIAL Y METODOS}

El estudio es de tipo descriptivo de corte transversal no experimental .la población se encuentra conformada por todos los estudiantes del VII ciclo de la Facultad de Enfermería de la Universidad Nacional San Luis Gonzaga de Ica, Perú, el tamaño de la muestra corresponde a 54 estudiantes de enfermería. La técnica utilizada para la recolección de datos fue la encuesta y el instrumento utilizado fue el cuestionario validado en Trujillo por la autora Sayre M. (10), el cual se restructuró a nuestra realidad, aplicando la prueba piloto al $15 \%$ de la muestra que permitió determinar las características finales del instrumento. La primera parte del instrumento que se administró señala la presentación, las instrucciones y datos generales. La segunda parte estuvo conformada por interrogantes para medir el nivel de conocimiento sobre reanimación cardiopulmonar. Se utilizó un cuestionario con escala vigesimal puntuándolas de (0-10 puntos) como deficiente de (11-15 puntos) regular y de (1620) como un nivel de conocimiento bueno, para el procesamiento de datos se utilizó la estadística descriptiva y el uso del software SPSS versión 22.

\section{RESULTADOS}

Se evidencio en los datos demográficos que el $90,74 \%$ (49 alumnos) son de sexo femenino y el 9,26\%(05 estudiantes) son de sexo masculino; el 46,30\%(25 estudiantes) de alumnos tienen 21 años, el 25,93\%(14 estudiantes) tienen 20 años, el 16.67\%(09 estudiantes) tienen 19 años y el 11,10\% (06 estudiantes) tienen de 22 a más años. En el Grafico 1 se analizó el Conocimiento sobre reanimación cardiopulmonar general obteniendo como resultados que un $48,15 \%$ de alumnos tienen los conocimientos deficientes sobre reanimación cardiopulmonar, el $44.44 \%$ tienen conocimiento regular y el $7,41 \%$ tienen conocimiento bueno en RCP básico y avanzado. Referente al Grafico 2. Conocimiento sobre reanimación cardiopulmonar básico El 46,30\% de alumnos tienen los conocimientos deficientes sobre reanimación cardiopulmonar básico, el 44,44\% tienen conocimiento regular y el $9,26 \%$ tienen conocimiento bueno. Con relación a Grafico 3. Conocimiento sobre reanimación cardiopulmonar avanzado se manifestó que El $50 \%$ de alumnos tienen los conocimientos deficientes sobre reanimación cardiopulmonar avanzado, el $46,30 \%$ tienen conocimiento regular y el $3,70 \%$ tienen conocimiento bueno.

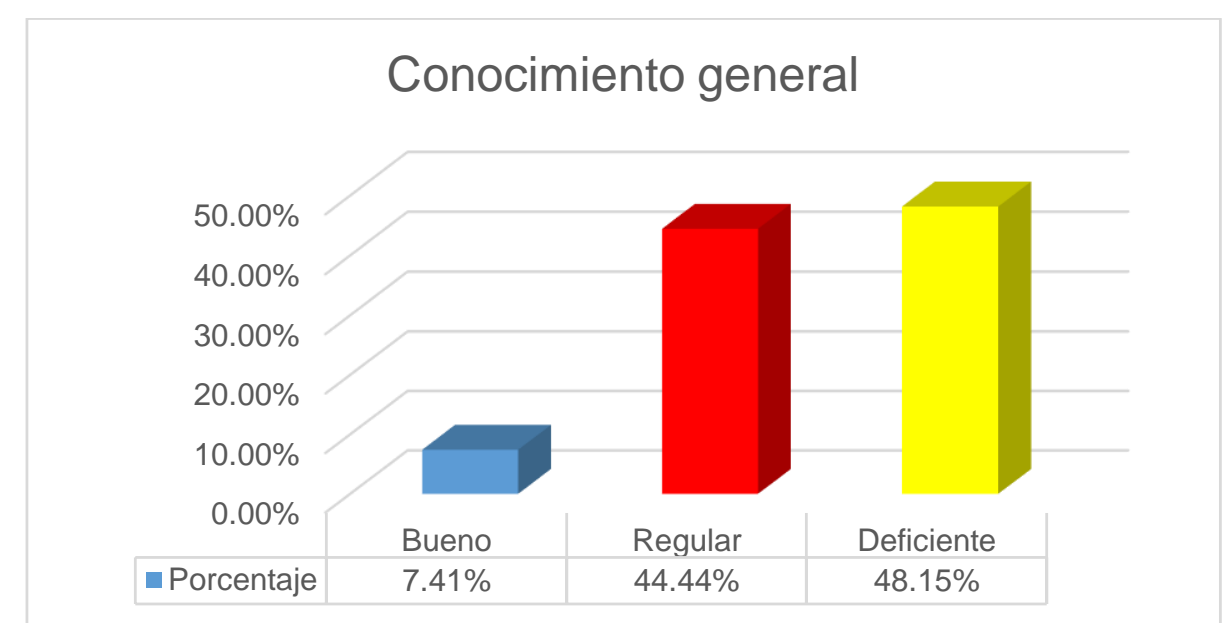

Gráfico 1: Conocimiento de los alumnos de VII ciclo encuestados sobre reanimación cardiopulmonar general 


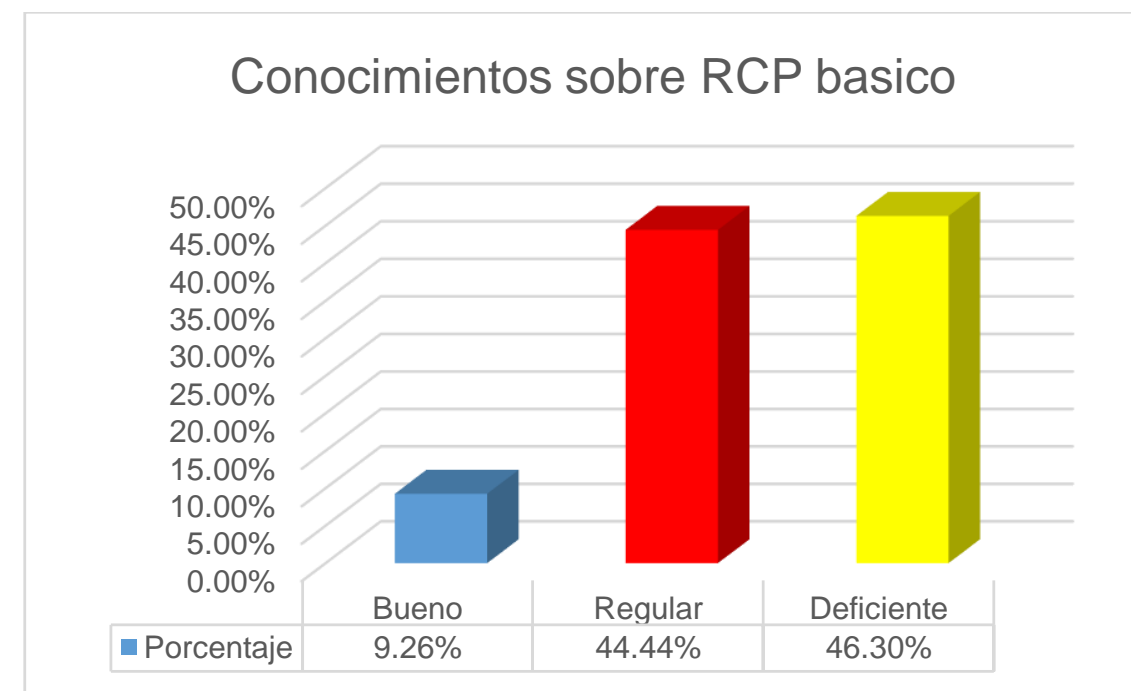

Gráfico 2: Conocimiento de los alumnos de VII ciclo encuestados sobre reanimación cardiopulmonar básico

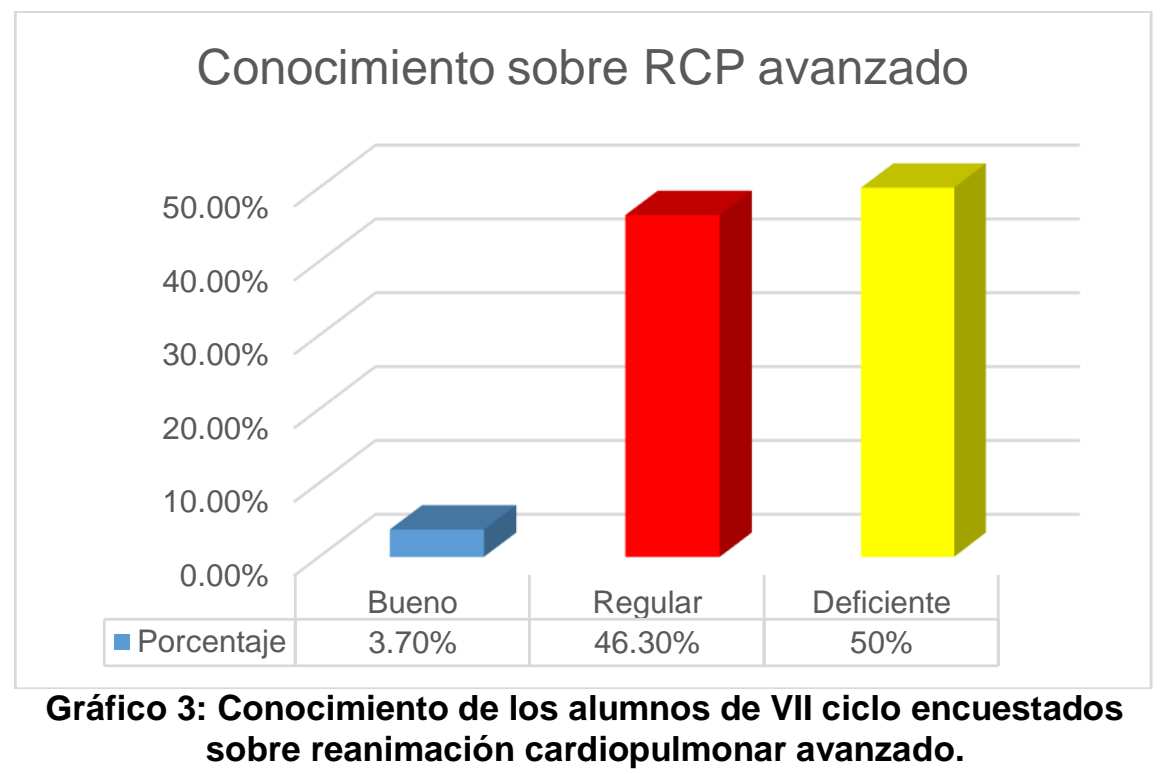

\section{DISCUSIÓN}

En el estudio se evidencio que el $46,30 \%$ de alumnos tienen los conocimientos deficientes sobre reanimación cardiopulmonar básico, el $44,44 \%$ tienen conocimientos regulares y el $9,26 \%$ tienen conocimiento bueno. El $50 \%$ de alumnos tienen los conocimientos deficientes reanimación cardiopulmonar avanzado, el $46,30 \%$ tienen conocimiento regular y el $3,70 \%$ tienen conocimiento bueno; siendo consistentes con: el estudio de Peralta J. Nivel cognitivo sobre reanimación cardiopulmonar básica en internos de un Hospital en Ecuador. Mostraron que un $75 \%$ tenían conocimientos bajo sobre RCP básico, un 44\% conocen como identificar un paro cardiorrespiratorio y un $66 \%$ saben sobre desfibrilación reportando tener un conocimiento medio (11). Arapa A. Nivel cognitivo sobre reanimación cardiopulmonar básica en internos de enfermería de la Universidad Nacional de Puno, Perú, los resultados obtenidos fueron que el $65 \%$ de los internos tenían nivel cognitivo bajo en RCP básico, mientras que un $35 \%$ tuvieron un nivel cognitivo medio y ninguno evidencio tener un conocimiento alto, en cuanto a los conocimiento de reanimación cardiopulmonar generales $80 \%$ tenía nivel cognitivo bueno, el $5 \%$ nivel cognitivo medio y el $15 \%$ nivel 
cognitivo bajo, respecto al reconocimiento de signos del paro cardiaco el $82,5 \%$ mostro un nivel cognitivo bajo mientras que $17,5 \%$ medio y ninguno con nivel cognitivo bueno, sobre los conocimientos de la realización de maniobras de reanimación un $60 \%$ obtuvo nivel cognitivo bajo el $35 \%$ medio y el $5 \%$ nivel cognitivo alto(7). Ballón F. (2016) Nivel cognitivo sobre reanimación cardiopulmonar (RCP) en internos de Medicina realizado en Arequipa, Perú. El $72,6 \%$ de los internos encuestados tenían un nivel cognitivo bajo, el $24,2 \%$ tiene nivel cognitivo regular, mientras que un $3,2 \%$ tuvo un nivel cognitivo bueno; se evidencio que existía una relación entre los internos que llevaron curso de RCP y sus conocimientos en comparación con los internos que no tenían ningún curso de RCP (8).

No siendo consistente con el estudio de Bueno C, Nivel de cognitivo sobre RCP en Internos de Medicina de la UPSJB en Lima, Perú .El 71\% tenía un conocimiento medio mientras el $16,1 \%$ tenían un conocimiento bajo y solo el $12,9 \%$ poseía un conocimiento alto en cuanto a los que conocen sobre reanimación cardiopulmonar básica el 64,5\% mostraba un conocimiento medio el $19,4 \%$ un alto nivel y el 16,1 bajo nivel y en conocimientos de reanimación cardiopulmonar avanzada se identificó que el $64,5 \%$ tenían nivel cognitivo medio un $19,4 \%$ bajo nivel y el $16,1 \%$ un alto nivel de estos datos. (6). Buscal P. Nivel de conocimiento y práctica simulada sobre Reanimación cardiopulmonar en los estudiantes del décimo ciclo de la Escuela TEZZA en Lima,Perú. Del 100\% de estudiantes del $X$ ciclo de la Escuela Tezza el $76 \%$ obtuvo un conocimiento regular sobre la RCP y el $72 \%$ presentó una práctica simulada adecuada sobre la RCP básica en adultos (12). Cárdenas J, Huamán C, Nivel cognitivo sobre reanimación cardiopulmonar en internos de medicina de Ica, Perú. Se registró que el $19,4 \%$ manifestaron que habían recibido un curso de RCP con respecto al conocimiento el $26,9 \%$ tenían conocimientos bueno, el 52,2\% obtuvo conocimientos medio y con un bajo nivel de conocimientos el 20,9\% (9).

Se llega a la conclusión que el mayor porcentaje de los profesionales de enfermería no tienen buenos conocimientos y la oportunidad de desarrollar capacidades en RCP básico y avanzado; por tal razón se requiere una capacitación y entrenamiento continuo en los estudiantes en el manejo inicial de las emergencias, actualizando las técnicas y fortaleciendo las destrezas, con el propósito de satisfacer la atención de salud desde el sitio donde ocurre la urgencia/emergencia.

\section{CONCLUSIONES}

1. Que el nivel de conocimientos sobre reanimación cardiopulmonar en forma global (reanimación cardiopulmonar básica y avanzada) en estudiantes de Enfermería del séptimo ciclo registraron mayoritariamente un nivel deficiente.

2. En cuanto a la reanimación cardiopulmonar básica. El mayor porcentaje de los estudiantes de la muestra de estudio manifestaron un nivel de conocimientos deficientes, registrándose una elevada proporción relacionada a las complicaciones que ocasiona realizar RCP, a la identificación de parocardiorespiratorio, y la secuencia de soporte vital, seguidamente con limitada diferencia un nivel de conocimientos regular mostrado significativamente referente a la relación entre compresiones torácicas y ventilaciones para RCP de adulto con uno o dos reanimaciones. $Y$ observándose una baja proporción del nivel de conocimiento bueno, respondiendo respecto a la profundidad de las comprensiones torácicas en el adulto.

3. Referente a la reanimación cardiopulmonar avanzada. los estudiantes manifestaron también nivel de conocimientos deficientes concerniente a lo que se debe realizar después de una desfibrilación en reanimación cardiopulmonar avanzado y en el paro cardio respiratorio por asistolia. 
Cuál es la intervención más útil. Luego registraron un conocimiento regular, respondiendo cifras considerables relacionada a la pregunta en una víctima de PCR por fibrilación ventricular cuál es el tratamiento más efectivo e ínfimo porcentaje tienen conocimiento bueno con solo $3.7 \%$. Cuando decimos que un ritmo cardiaco es desfribrilable.

\section{CORRESPONDENCIA}

Lic. en Enf. Yuri Katherine Miranda Conislla

Correo electrónico:

yurimiranda_26@outlook.com

\section{REFERENCIAS BIBLIOGRAFICAS}

1. Monzón J L, Saralegui I, Molina R, Abizanda R, Cruz Martín M, Cafré L, et al. Ética en las maniobras de resucitación cardiopulmonar. Lima, Perú Rev. Med Intensiva. 2015; 34 (6):534-549.

2. O’Connor R. Manual MSD Versión para profesionales. Reanimación Cardiopulmonar RCP) en adultos. Estados Unidos; 2019

3. American Heard Association.Marque la diferencia con un ser querido, 2019. (Internet).Estados Unidos ; 2019.(Citado 15 marzo 2019).Disponible en: https://international.heart.org/es/ourcourses/be-difference-someone-you-love

4. American Heard Association.RCP usando solo las manos,05/2019 (Internet). Estados Unidos; 2019. (Citado 10 marzo 2019). Disponible en :https://www.ilcor.org/data/DS14700 CPRWeek FactSheet-Spanish.pdf

5. Balcázar L. Nivel cognitivo sobre reanimación cardiopulmonar en personal de urgencias 2016. Rev. Esp Med Quir; 20 (2). 248-255.
6. Bueno C. Nivel de Cognitivo sobre RCP en Internos de Medicina de la UPSJ; Lima; 2018. (Internet). Lima, Perú; 2018. (Citado el 10 marzo. 2019).Disponible en: http://repositorio.upsjb.edu.pe/bitstream/h andle/upsjb/1498/T-TPMC\%20Cindy\%20Janina\%20\%20Bueno\%20 Mallma.pdf?sequence $=1$ \&isAllowed $=y$

7. Arapa A. Nivel cognitivo sobre reanimación cardiopulmonar básica en internos de enfermería de la universidad Nacional, Puno; 2017. (Internet) Puno, Perú; 2017. (Citado el 10 marzo. 2019). Disponible en: http://repositorio.unap.edu.pe/bitstream/ha ndle/UNAP/6329/Arapa_Quispe_Ana_Mar ia.pdf?sequence $=1$ \& isAllowed $=y$

8. Ballon F. Nivel de Conocimiento Sobre Reanimación Cardiopulmonar (RCP) en Internos de medicina Humana, Arequipa 2016.(Internet). Arequipa, Perú; 2016. (Citado 5 de Enero del 2019) disponible en: http://alicia.concytec.gob.pe/vufind/Record IUCSM 0e9d3ffa91f5 288542fa6947687819a9/Details.

9. Cárdenas J, Huamán C. Nivel de conocimiento sobre reanimación cardiopulmonar en internos de medicina de la provincia de Ica. 2016. Rev. Médica Panacea. Ica, Perú 2017: 5 (3). (En línea) (Citado el 10 de marzo. 2019). Disponible en:

$<$ http://revpanacea.unica.edu.pe/index.ph p/RMP/article/view/62>

10. Sayre M. Reanimación Cardiopulmonar y Paro Respiratorio. (Internet). Trujillo, Perú; 2016 (Citado el 10 de abril 2019) Disponible en: http://reanimacioncardiopulmonar.blogspot .pe/2007/12/cuidados-de-enfermeraenreanimacin.html. 
11. Peralta J. Nivel cognitivo sobre reanimación cardiopulmonar básica en internos de un Hospital. Ecuador; 2016. (Internet). Ecuador;2016 (Citado el 5 de enero 2019) Recuperado a partir de: http://dspace.uniandes.edu.ec/handle/123 456789/5152.
12. Buscal P. Nivel de conocimiento y práctica simulada sobre reanimación cardiopulmonar en los estudiantes del décimo ciclo de la escuela Tezza.Lima,2019. (Internet). Lima, Perú; 2019. (Citado el 14 Noviembre 2019) Disponible en: http://repositorio.urp.edu.pe/bitstream/han dle/urp/2613/tesis\%20tezza\%20final\%202 019.pdf? sequence $=1$ \&isallowed $=y$

Recibido:14/07/2021

Aprobado para publicación: 12/10/2021 